\title{
Mulheres em situação de violência: entre rotas críticas e redes intersetoriais de atenção
}

\author{
Violence against women: between critical path and \\ assistance multisectoral networks
}

\section{Ana Flávia Pires Lucas D’Oliveira ${ }^{1}$, Lilia Blima Schraiber ${ }^{1}$}

D’Oliveira AFPL, Schraiber LB. Mulheres em situação de violência: entre rotas críticas e redes intersetoriais de atenção / Violence against women: between critical path and assistance multisectoral networks. Rev Med (São Paulo). 2013 abr.-jun.;92(2):134-40.

RESUMO: A violência contra a mulher é um problema de alta prevalência e grande impacto nas condições de saúde de mulheres e crianças. $\mathrm{O}$ artigo aborda como o tema da violência doméstica e sexual contra a mulher assume, nas últimas décadas, o caráter de problema de saúde pública e direitos humanos. Baseando-se em revisão da literatura, retrata-se uma breve história das políticas públicas dirigidas ao tema e discute-se dois conceitos interligados: a rota crítica das mulheres afetadas pela violência doméstica (como as mulheres lidam com a violência e as dificuldades encontradas) e a necessidade de uma rede intersetorial de atenção. As possibilidades de integração dos serviços de saúde nesta rede intersetorial é tematizada. Conclui-se com a necessidade do reconhecimento por todos os profissionais da rede dos eixos de desigualdade social, especialmente no que tange às diversas manifestações das desigualdades de gênero, mas também de classe e raça/etnia ${ }^{28,29}$, para efetiva proteção dos direitos humanos e sociais e o trabalho com a violência nos serviços de saúde. Levar em consideração esse aspecto é extremamente importante, e propõese aos profissionais de saúde uma abertura ao diálogo acerca de alternativas para a ação que, ao tomar a violência como questão também a ser enfrentada nos serviços de saúde, possa representar igualmente uma ampliação do espectro tecnológico com maior satisfação no trabalho cotidiano.

DESCRITORES: Mulheres; Saúde da mulher; Violência contra a mulher; Violência doméstica; Ação intersetorial.

\begin{abstract}
Violence against women is a problem with high prevalence and huge impact in women and children's health conditions. This paper approaches how the issue of domestic and sexual violence against women has been treated, in the last decades, as a human rights and public health problem. Based in a literature review, a brief history of the public policies to the problem in Brazil is depictured. Two interconnected concepts are discussed: the critical path of women experiencing domestic violence (how women cope with violence and the difficulties that they face) and the need of a multisectoral assistance network. The possibilities of inclusion of health care facilities in this multisectoral network are discussed. We finalize highlighting the need of the recognition by all professionals in the network of the different axis of social iniquity, specially related to gender iniquities but also class and race ones. To consider this aspect is essential, and we invite health workers to open to dialogue about the different options of action. To face violence as an issue to be approached also by health services may represent a broadening of the technological scope with more satisfaction of the professionals with their work.
\end{abstract}

KEYWORDS: Women; Women's health; Violence against women; Domestic violence; Intersectorial action.

\footnotetext{
1. Professoras do Departamento de Medicina Preventiva da Faculdade de Medicina da Universidade de São Paulo - FMUSP. Endereço para correspondência: Departamento de Medicina Preventiva da FMUSP. av. Dr. Arnaldo, 455 - São Paulo, SP. e-mail: vawbr@gmail.com, aflolive@usp.br, liliabli@usp.br
} 
$\mathbf{T}$ em sido crescente o interesse pela questão da violência em interconexão com agravos à saúde. Para além do impacto já bem estudado das agressões e suicídios como parte das mortes violentas, sobretudo nas faixas etárias de jovens do sexo masculino, e que também estão relacionadas a questões raciais ${ }^{1}$, muitas são as publicações que apontam para a violência não fatal e seu impacto na saúde ${ }^{2}$. Destacam-se nesse sentido a situação das mulheres, cuja forma predominante de vivenciar as violências são as do tipo doméstico e em geral perpetradas pelos parceiros íntimos ${ }^{3,4,5}$. São situações reconhecidamente de longa duração e que produzem um leque amplo de danos à saúde das próprias mulheres, tais como uma maior chance de apresentarem pior avaliação de sua saúde e queixas de dor, perda de memória, tontura e corrimento vaginal ${ }^{6}$ transtornos mentais comuns ${ }^{7}$, ideação suicida $^{8}$, abortamentos, infecções por DST/HIV, gravidez indesejada e disfunções sexuais, além de patologias como fibromialgia ${ }^{3,9}$. Todas essas situações fazem com que estas mulheres usem com maior frequencia os serviços de saúde $^{10}$, embora com pouca adesão a medidas preventivas, como uso de condom ${ }^{3}$. A violência doméstica contra a mulher também ocasiona danos a seus filhos, tais como menor imunização infantil, maior mortalidade até os cinco anos $^{11}$ e maior prevalência de comportamento agressivo e problemas escolares ${ }^{12}$. As crianças, além de testemunhar a violência, podem também ser agredidas pelo parceiro ou pela própria mulher, ocupando tais mulheres muitas vezes a situação de vítimas de seus parceiros e de agressoras de seus filhos ${ }^{2}$.

Em razão dessa grande repercussão na saúde, as violências experimentadas pelas mulheres deveriam ser abordadas pelos serviços que as atendem. No entanto, sem contar com o desenvolvimento de uma assistência específica e integrada ao atendimento de rotina nas unidades de saúde, quem vive ou viveu situações de violência não encontra atendimento apropriado. Este implicaria um modo específico de acolhimento, porque essa violência vivida não é facilmente relatada pelas mulheres, fenômeno que tem sido trabalhado na literatura como a 'invisibilidade' da violência no campo da Saúde ${ }^{13,14}$. Também implicaria um modo específico de ouvir e trabalhar a situação relatada com as mulheres, como uma proposta assistencial compatível com o enfrentamento direto do problema, como discutiremos a seguir.

Sem ser uma questão da competência exclusiva da Saúde, a violência contra a mulher é de tal magnitude que se postula, internacionalmente, seu reconhecimento como problemática da Saúde Pública ${ }^{2}$. De fato, isto representaria um avanço necessário na direção da promoção da saúde e uma definitiva posição do setor quanto a seu compromisso com os Direitos Humanos. Tal atitude permitiria tornar claro a adoção do princípio da não-violência como direito e como valor ético para a política de saúde, com suportes substantivos para a ampliação dos direitos das mulheres, incluindo-se os direitos reprodutivos já assumidos em programas aprovados pelo Ministério da Saúde e as Secretarias Estaduais e Municipais de Saúde.

Não obstante, sabemos que os profissionais nos serviços de saúde têm dificuldades para lidar com problemas como a violência, que são problemas mais identificados ao social e às normas culturais da sociedade ou identificados a comportamentos pessoais. São dificuldades que não se prendem apenas ao quê fazer, dentro de um leque de atuações bem delimitadas como intervenções sobre as doenças, mas dizem respeito, previamente, ao reconhecimento dessa situação de violência como pertinente ao campo de atuação em saúde.

Os profissionais da saúde lidam mal com demandas que nem sempre se caracterizam como adoecimentos, ainda que constituam, de fato, sofrimentos com potenciais danos à saúde. Nos serviços de atenção primária, em prontoatendimentos ou mesmo em prontos-socorros, muitas dessas situações representam verdadeiros obstáculos comunicacionais, já que são expressas na forma de múltiplas queixas, dores de imprecisa localização no corpo ou sem correspondência a patologias conhecidas. Sendo muitas vezes situações diagnósticas imprecisas, as demandas trazidas pelas mulheres que estão vivendo situações de violência com frequência são assumidas como situações assistenciais fadadas ao insucesso. Do ponto de vista dos serviços de saúde, portanto, há que se pensar em uma reorientação gerencial e técnica das práticas exercidas por seus profissionais. E essa reorientação tem sido tanto mais urgente quanto mais os diversos setores da sociedade brasileira que respondem por assistência à população, em termos de sua saúde, segurança e realização de direitos, estiverem comprometidos com as políticas públicas já promulgadas e que postulam serviços de atenção a vítimas de violência e de prevenção dessa ocorrência.

Durante a década de 1980, a partir da convergência do movimento social de mulheres e do feminismo, que exercem pressões sobre o Estado para que haja investimentos em Políticas Públicas com recorte de gênero, foram criados os primeiros serviços específicos para a atenção a mulheres em situação de violência ${ }^{15}$, as Delegacias de Defesa das Mulheres. Nas últimas três décadas, assistimos no Brasil a um grande aumento desses serviços, ao lado de intenso movimento pelos direitos humanos das mulheres, do incremento na produção de conhecimento científico sobre o tema e da assinatura pelo país de diversas Conferências Internacionais comprometendo-se a combater a violência contra a mulher, como a Convenção para a Eliminação de todas as formas de discriminação contra as mulheres (1979), a Conferência Internacional sobre Direitos Humanos - Viena (1993), a Conferência Internacional sobre População e Desenvolvimento - Cairo (1994), a Convenção Interamericana para Prevenir, Punir e Erradicar a Violência Contra a Mulher - Belém do Pará (OEA) (1994) e a $4^{\mathrm{a}}$ Conferência Mundial sobre a Mulher - Beijing (2005). 
Houve também o surgimento de Conselhos da Condição da Mulher e das Coordenadorias da Mulher em administrações estaduais e municipais e, em 2003, da Secretaria de Políticas para Mulheres no governo federal ${ }^{15}$.

Em 2006 é promulgada a Lei 11.340, conhecida como Lei Maria da Penha, resultado da mobilização realizada nas décadas anteriores e inspirada na Convenção Interamericana para Prevenir, Punir e Erradicar a Violência contra a Mulher. Além de tipificar o crime de violência doméstica e familiar contra a mulher, a Lei Maria da Penha prevê diversas medidas e serviços para o trabalho com o problema, estabelecendo a necessidade do trabalho articulado de diversos setores sociais para o seu enfrentamento, inclusive da saúde. A Lei ainda prevê a necessidade de capacitação permanente dos profissionais trabalhadores dos diversos setores envolvidos em questões de gênero e de raça ou etnia.

Em 2007, foi lançado pelo Presidente da República do Brasil o Pacto Nacional para o Enfrentamento da Violência contra a Mulher ${ }^{16}$, como parte da Agenda Social do Governo Federal, que consiste num acordo federativo entre o governo federal, os governos dos estados e os municípios brasileiros para o planejamento de ações que visem à consolidação da Política Nacional de Enfrentamento à Violência contra as Mulheres por meio da implementação de políticas públicas integradas em todo território nacional ${ }^{17}$.

Além disto, na área da saúde, em 1999, é lançada pelo Ministério da Saúde a Norma Técnica para a prevenção e tratamento dos agravos resultantes da violência sexual contra mulheres ${ }^{18}$ que estabelece um protocolo de atenção à violência sexual para mulheres e meninas, detalhando a profilaxia de DST, medicação antirretroviral, gravidez indesejada e o apoio psicossocial e em 2003 a violência contra a mulher torna-se de notificação compulsória para a vigilância epidemiológica ${ }^{17}$.

De outro lado, é importante destacar que a literatura assinala que a busca de apoio no enfrentamento dessas situações por parte das mulheres ainda é repleta de dificuldades subjetivas e obstáculos objetivos na dinâmica da vida social, comunitária, familiar e dos relacionamentos de casal $^{19}$

Assim, nosso propósito no presente texto é o de cotejar esses dois lados da questão: como as mulheres enfrentam o problema buscando apoios e como os serviços têm atuado como apoio social institucionalizado.

\section{A rota crítica das mulheres no enfrentamento da violência}

Por que as mulheres permanecem nas situações de violência conjugal é uma pergunta recorrente nas práticas assistenciais e na pesquisa sobre o tema. Quando colocamos a questão nesses termos, estamos tacitamente assumindo que permanecer na relação violenta é uma escolha livre da mulher, independente do contexto em que ela se encontra. Obscurecemos, assim, toda rede de relações de cada indivíduo particular, e a importância, nas decisão e ações de cada um, de suas relações pessoais próximas, e dos recursos comunitários e sociais presentes, incluindo o universo cultural em que se está imerso.

Em verdade, pesquisas têm demonstrado que mulheres em situação de violência conjugal buscam diversas alternativas para superar a sua situação, sendo bastante ativas no sentido de buscar interromper a violência, sempre que possível.

Ao invés de manter silêncio, a grande maioria das mulheres que vivem ou viveram situações de violência física por parceiro no município de São Paulo $(73,9 \%)$ ou a Zona da Mata do Recife (69,3\%) conversaram sobre o seu problema com alguém e pediram ajuda. O pedido de ajuda à rede informal, formada por família e amigos foi bem mais frequente do que o pedido à rede formal de serviços, que foi de $33,8 \%$ em São Paulo e apenas $17,1 \%$ na Zona da Mata de Pernambuco. As razões relatadas pelas mulheres para a busca de serviços para combater a violência foram majoritariamente "não aguentava mais" e "muito machucada, medo de que ele a matasse" $"$. Por outro lado, as que não buscaram serviços justificaram de forma mais frequente não terem procurado ajuda formal dizendo que "a violência é normal", ou seja, banalizando a violência.

Coerentemente, quanto mais grave a violência, maior a procura por apoio, o que faz com que os casos que acorrem aos serviços especializados sejam já bastante graves. No entanto, buscar apoio não significa necessariamente encontrá-lo. Em São Paulo, 59,2\% das mulheres estudadas encontraram algum apoio em amigo e família e em ZMP 56,4\% ${ }^{20}$

Pesquisa realizada na América Latina pela Organização Panamericana de Saúde ${ }^{19}$ e replicada no Brasil $^{21}$ demonstra como a busca das mulheres afetadas pela violência intrafamiliar pode ser difícil e cheia de obstáculos. As mulheres encontram respostas dissuadoras de seu projeto de interromper a violência por parte de familiares, comunidades e serviços estatais e não-governamentais como delegacias de polícia, serviços jurídicos, de saúde e assistência social. Informações inadequadas e atitudes não acolhedoras e crivadas de julgamento moral são frequentes nos percursos relatados nas investigações ${ }^{19,21,22}$.

Por isso, Sagot ${ }^{19}$ cunhou este percurso como uma "rota crítica", já que as decisões e ações das mulheres para interromper o ciclo da violência, ao receberem respostas inadequadas ou culpabilizadoras, acabam por não ser efetivas, reiterando a violência.

Se as respostas inadequadas da comunidade e dos profissionais dos serviços têm grande importância na rota crítica, é essencial examinarmos os obstáculos colocados para que esses profissionais possam atuar de forma mais efetiva, visando a garantia dos direitos das mulheres 
articulada, no caso dos serviços de saúde, com a garantia à sua saúde.

\section{A saúde e a rede interssetorial de serviços}

Apesar do avanço representado pelas iniciativas legislativas, de políticas públicas e de organização e distribuição social de serviços voltados especificamente à violência contra a mulher (como Delegacias de Defesa da Mulher, abrigos, serviços de atendimento à violência sexual, defensorias da mulher, Varas especiais para violência doméstica e intra-familiar, centros de referência etc.), muitas dificuldades ainda persistem no que diz respeito tanto à qualidade da assistência oferecida em cada serviço e a capacidade de bem responder à busca por ajuda por parte das mulheres em suas rotas para lidar com a violência que experimentam, quanto à articulação dos serviços entre si. Esses serviços fazem parte de dispositivos governamentais e não governamentais, e se distribuem em diversos setores de prestação de assistência, compondo um grande mosaico, mas ainda pouco inter-relacionado.

Os estudos realizados sobre a oferta de cuidado pelos serviços criados especificamente para trabalhar com o problema da violência de gênero têm mostrado muitas dificuldades na operação dos princípios contidos nas Leis e políticas conquistadas. Os atendimentos são marcados por ambiguidades e contradições, os espaços e fluxos são inadequados à tarefa e a percepção dos profissionais é permeada, muitas vezes, por estereótipos acerca das relações de gênero, como mostra um estudo realizado em serviços de saúde e Delegacias de Defesa da Mulher ${ }^{22}$. Além disto, a lógica dos profissionais e suas expectativas quanto ao trabalho a ser realizado muitas vezes desconhece ou interpreta parcial ou equivocadamente as necessidades expressas pelas mulheres e as possibilidades de intervenção que elas almejam, criando obstáculos para a efetivação dos direitos em locais criados para garanti-los, como as Delegacias de Defesa da Mulher ${ }^{23,24}$.

Para que esses serviços possam trabalhar de forma articulada, é necessária a sua articulação em rede assistencial, como coloca a própria Secretaria de Políticas para Mulheres. Genericamente, redes referem-se ao estudo não apenas de objetos singulares, mas especialmente das relações entre eles ${ }^{25,26}$. Por essas relações, ou fluxos, circulam ideias, sujeitos e coisas. Consideraremos neste artigo, baseados em teorias acerca do trabalho em equipe na área da $\mathrm{Saúde}^{27}$, o estabelecimento de uma rede de serviços como dependente da articulação das ações e interação dos agentes do trabalho para possibilitar o estabelecimento de um projeto assistencial comum para os casos que se apresentam, em que cada profissional e cada setor assistencial tem uma contribuição parcelar, específica mas interdependente e todos se orientam pela defesa dos direitos humanos e sociais e o estabelecimento de relações interpessoais não violentas como norte ético e político do trabalho ${ }^{28}$.

O estabelecimento dessa rede necessita do reconhecimento recíproco entre os diversos setores assistenciais, nas suas especificidades e também no que tenham em comum, ou seja, serem parte de uma rede de proteção de direitos. Para isso, há que superar a visão restrita e independente de cada disciplina, profissão, serviço e setor assistencial sobre o problema, e há que reconhecer as mulheres como cidadãs, titulares de direitos e capazes de escolhas autônomas sobre suas vidas. Por outro lado, para a efetiva proteção dos direitos, é necessário o reconhecimento por todos dos eixos de desigualdade social, especialmente, no que tange às diversas manifestações das desigualdades de gênero, mas também de classe e raça/etnia ${ }^{28,29}$.

E como os serviços de saúde podem ou devem se articular a uma rede assim estabelecida? Uma revisão das estratégias para lidar com essa forma de violência nos serviços de saúde em países de renda média ou baixa descreve iniciativas emergentes para o trabalho com violência de gênero em serviços de saúde primários, secundários e terciários. A articulação, entretanto, seja interna à saúde, seja com a rede intersetorial, ainda é bastante rara e incipiente. Em resumo, se é consenso que os serviços de saúde devem tornar a violência doméstica visível e trabalhar o problema no seu interior, quais ações e programas devem ser criados ainda não estão bem definidos ${ }^{30}$, e como devem se articular em rede tampouco é algo já bem estabelecido.

No Brasil, os primeiros serviços de saúde a trabalhar com a violência contra a mulher foram os hospitais que implantaram o atendimento à violência sexual, com o oferecimento de profilaxia para DST/HIV e aborto legal se necessário. Hoje, já são 443 instituições que realizam atendimento à mulheres vítimas de violência sexual em todo o território nacional ${ }^{17}$. No entanto, esses serviços são concentrados em hospitais e em cidades maiores, e acabam por atender basicamente a violência sexual cometida por estranhos, mantendo na invisibilidade a violência sexual perpetrada por parceiro íntimo.

Os serviços de atenção primária têm importantes especificidades nesse debate: por um lado, estão em contato com a grande maioria da população e poderiam ser uma chance de detecção precoce dos casos, anterior à busca de serviços hospitalares ou Delegacias de Polícia por danos mais graves. Por outro lado, com a implantação da Estratégia de Saúde da Família no Brasil, os Agentes Comunitários de Saúde e os profissionais das Unidades Básicas de Saúde em geral estão mais próximos dos domicílios, entrando em maior contato com casos de violência doméstica. Existem já estímulos e programas para que o tema seja abordado na rede básica de saúde ${ }^{31}$, e a violência contra crianças e contra mulheres deve ser compulsoriamente notificada à vigilância epidemiológica. No entanto, as ações ainda são 
isoladas, fragmentadas e em caráter pessoal, e há grande dificuldade em reconhecer o trabalho com a violência doméstica de gênero como do escopo dos profissionais de saúde $^{32}$, e resistências quanto à notificação ${ }^{33}$.

Os profissionais, treinados para detectar patologias, encontram-se frente a um tema que, embora impacte fortemente nos padrões de saúde-doença das populações, não é uma patologia, e necessita, para seu enfrentamento, de treinamento e supervisão que trate de como conversar sobre o assunto, como lidar com seus próprios valores e como incluir questões de gênero na prática assistencial, além de conhecimento da rede interssetorial pertinente.

Para que a revelação dos casos se dê, é necessário que os profissionais ajam com uma atitude interessada e não julgadora, com um atitude clara de não vitimização e apoio à mulher. Mas, embora muito importante, a escuta , qualificada e acolhedora, tomando o tema como importante para o trabalho em saúde não é o bastante. É necessário situar as demandas e oferecer alternativas assistenciais à mulher e à sua família/rede no interior dos serviços de saúde (contracepção adequada, psicoterapia, atendimento a abuso de substâncias, grupos) assim como em outros setores assistenciais (Delegacias das Mulheres, Defensorias da Mulher, Centros de Referência, Serviços de assistência social, trabalho e moradia). Para isso, é necessário haver o reconhecimento prévio da rede de serviços, que, em São Paulo, está no site (www.rededecidadania.com.br), e, no Brasil, pode ser encontrada no site da Secretaria de Políticas para as Mulheres ${ }^{34}$. Conhecer esses equipamentos é muito importante para que possam ser criados laços mais estreitos de referência e contrarreferência que possam trabalhar no sentido do estabelecimento de projetos assistenciais comuns. Dessa forma, em decisão compartilhada com as mulheres, os profissionais podem apoiá-las no percurso de sua rota, transformando-nas em rotas menos críticas e mais efetivas na garantia dos direitos humanos e sociais envolvidos ${ }^{35}$.

Esse posicionamento ético e político claro contrário à violência tem um sentido técnico imbricado: deixar claras as consequências da violência para a saúde e apoiar as mulheres integralmente nas suas necessidades produz saúde, evita reduzir a queixas e patologias todos os sofrimentos e redireciona uma demanda frequente nos serviços de saúde de atenção primária e emergências para uma rede interssetorial em que elas possam ser atendidas em suas necessidades com maior propriedade, mantendo o vínculo e a longitudinalidade da atenção na saúde.

Essas questões nos levam a compreender as dúvidas expressas pelos profissionais da saúde como dúvidas genuínas, quanto a atuarem ou não sobre a violência. A noção de genuinidade pautada, aqui, na possibilidade/ impossibilidade de um agir comunicativo, significa nosso entendimento de que os profissionais falam/atuam desde o seu lugar de agentes de uma dada técnica e sujeitos portadores de uma dada cultura profissional. Seu agir, neste caso, estará conforme com esta cultura, ética e tecnicamente. Além do que, é relevante se considerar a peculiaridade da prática médica, a que se pode caracterizar como uma técnica moraldependente, em razão do entrelaçamento de sua intervenção técnica com a relação interindividual construída a cada ato, substantivamente entrelaçando a dimensão de trabalho com a interação humana nesta assistência. Assim, o componente ético estará, de fato, construindo a intervenção, desde o momento diagnóstico, o julgamento clínico e as propostas terapêuticas, conceba-o ou não o profissional em exercício. Sendo esse aspecto em grande medida extensivo às ações da assistência individual no cuidado em saúde de modo geral, um agir voltado para reconhecer e lidar com os direitos das mulheres, de imediato, revelaria às usuárias atendidas nos serviços a ética da não violência, de modo interno ao saber autorizado e legitimado exercido pelos profissionais. Levar em consideração esse aspecto é extremamente importante, e pretende ser um convite aos profissionais de saúde, uma abertura ao diálogo acerca de alternativas para a ação que, ao tomar a violência como questão também a ser enfrentada nos serviços de saúde, possa representar igualmente uma ampliação do espectro tecnológico com maior satisfação no trabalho cotidiano.

\section{REFERÊNCIAS}

1. Schraiber LB, D'Oliveira AFPL, Couto MT. Violência e saúde: estudos científicos recentes. Rev Saúde Pública. 2006;40:11220. http://dx.doi.org/10.1590/S0034-89102006000400016.

2. Krug EG, Dahlber LL, Mercy JA, Zwi AB, Lozano R. World report on violence and health. Genebra: World Health Organization; 2002. Available from: http://www.who.int/ violence_injury_prevention/violence/world_report/en/ summary_en.pdf

3. Heise L, Garcia-Moreno C. Intimate partner violence. In: Krug EG, Dahlber LL, Mercy JA, Zwi AB, Lozano R. World report on violence and health. Genebra: World Health Organization; 2002. p. $87-121$

4. Jewkes R, Sen P, Garcia-Moreno C. Sexual violence. In: Krug EG, Dahlber LL, Mercy JA, ZWI AB, Lozano R. World report on violence and health. Genebra: World Health Organization; 2002. p. 147-82.

5. Schraiber LB, D'Oliveira AFPL, Couto MT, Hanada H, Kiss LB, Durand J, et al. Violência contra a mulher entre usuárias de serviços básicos de saúde da rede pública da grande São Paulo. Rev Saúde Pública. 2007;41(3):359-67. http://dx.doi. 


\section{org/10.1590/S0034-89102007000300006}

6. Ellsberg M, Jansen HAFM, Heise L, Watts CH, GarciaMoreno C. Intimate partner violence and women's physical and mental health in the WHO multi-country study on women's health and domestic violence: an observational study. Lancet. 2008;371:1165-72. doi: 10.1016/S01406736(08)60522-X.

7. Ludermir AB, Schraiber LB, D’Oliveira AFPL, França-Junior I. Violence against women by their intimate partner and common mental disorders. Social Sci Med. 2008;66:1008-18. doi: 10.1016/j.socscimed.2007.10.021.

8. Devries K, Watts CH, Yoshihama M, Kiss LB, Schraiber LB, Deyessa N, Heise L, Durand JG, Mbwambo J, Jansen HAFM, Berhane Y, Ellsberg M, Garcia-Moreno C. Violence against women is strongly associated with suicide attempts: evidence from the WHO multi-country study on women's health and domestic violence against women. Social Sci Med. 2011;73(1):79-86. doi: 10.1016/j.socscimed.2011.05.006.

9. Barros CRS, Schraiber LB, França-Junior I. Associação entre violência por parceiro íntimo contra a mulher e infecção por HIV. Rev Saúde Pública. 2011;45:365-72. http://dx.doi. org/10.1590/S0034-89102011005000008

10. Schraiber LB, Barros CRS, Castilho EA. Violência contra as mulheres por parceiros íntimos: usos de serviços de saúde. Rev Bras Epidemiol. 2010;13(2):237-45. http://dx.doi. org/10.1590/S1415-790X2010000200006

11. Ellsberg M, Pena R, Herrera A, Liljestrand J, Winkvist A. Candies in hell: women's experiences of violence in Nicaragua. Social Sci Med. 2000;51(11):1595-610. http:// dx.doi.org/10.1016/S0277-9536(00)00056-3

12. Durand J, Schraiber LB, França Jr I, Barros C. Repercussão da exposição à violência por parceiro íntimo no comportamento dos filhos. Rev Saúde Pública. 2011;45(2):355-64. http:// dx.doi.org/10.1590/S0034-89102011005000004

13. Schraiber LB, D'Oliveira AFPL, Hanada H, Figueiredo WS, Couto MT, Kiss LB, Durand JG, Pinho AA. Violência vivida: a dor que não tem nome. Interface. Comun Saúde Educ. 2003;7(12):41-54. http://dx.doi.org/10.1590/S141432832003000100004 .

14. Schraiber LB, D’Oliveira AFPL. Violência contra mulheres: interfaces com a saúde. Interface Comun Saúde Educ. 1999;3:11-27. Disponível em: http://www.scielo.br/pdf/icse/ v3n5/03.pdf

15. Bandeira L. Três décadas de resistência feminista contra o sexismo e a violência feminina no Brasil: 1976 a 2006. Sociedade e Estado, Brasília. 2009;24(2):401-38. Disponível em: http://www.scielo.br/pdf/se/v24n2/04.pdf

16. Brasil. Secretaria de Políticas para as Mulheres. Política nacional de enfrentamento à violência contra as mulheres. Brasília; 2011. Disponível em: https://sistema3.planalto.gov. $\mathrm{br} / / \mathrm{spmu} /$ atendimento/atendimento_mulher.php

17. Barsted LL, Pitanguy J, organizadores. O progresso das mulheres no Brasil 2003-2010. Rio de Janeiro: CEPIA; ONU Mulheres; 2011. Disponível em: http://www.unifem.org.br/ sites/700/710/progresso.pdf

18. Brasil. Ministério da Saúde. Área Técnica de Saúde da Mulher. Prevenção e tratamento dos agravos resultantes da violência sexual contra mulheres e adolescentes: norma técnica. 2a ed. Brasília; 2005. Disponível em: http://bvsms.saude.gov.br/bvs/ publicacoes/caderno6_saude_mulher.pdf

19. Sagot M. Ruta crítica de las mujeres afectadas por la violencia intrafamiliar en América Latina: estudios de caso de diez países. Washington: PAHO; 2000. Disponível em: http:// ns.bvs.hn/docum/ops/libros/rutacritica.pdf

20. Kiss LB, D’Oliveira AFPL, Zimmerman C, Heise L, Schraiber $\mathrm{LB}$, Watts $\mathrm{CH}$. Brazilian policy responses to violence against women: government strategy and the help-seeking behaviors of women who experience violence. Health Human Rights. 2012;14:1-14. Available from: http://researchonline.lshtm. ac.uk/24381/2/Kiss-FINAL2.pdf

21. Meneghel SN, Bairros F, Mueller B, Monteiro R, Oliveira LP, Collaziol ME. Rotas críticas de mulheres em situação de violência: depoimentos de mulheres e operadores em Porto Alegre, Rio Grande do Sul, Brasil. Cad Saúde Pública. 2011;27:743-52. http://dx.doi.org/10.1590/S0102311X2011000400013

22. Villela WV, Vianna LA, Lima LF, Sala DCP, Vieira TF, Vieira ML, Oliveira EM. Ambiguidades e contradições no atendimento de mulheres que sofrem violência. Saúde Soc. 2011;20(1):113-23. http://dx.doi.org/10.1590/S010412902011000100014

23. Brandão ER. Violência conjugal e o recurso feminino à polícia. In: Bruschini C, Hollanda HB, organizadores. Horizontes plurais: novos estudos de gênero no Brasil. São Paulo: Fundação Carlos Chagas/Editora 34; 1998. p.51-84.

24. Campos CH. Justiça consensual, violência doméstica e direitos humanos. In: Strey MN, Azambuja MPR, Jaeger FP. Violência, gênero e políticas públicas. Porto Alegre: EDIPUCRS; 2004. p.63-84.

25. Marteleto RM, Silva ABO. Redes e capital social: o enfoque da informação para o desenvolvimento local. Ci Inf(Brasília). 2004;32(3):41-9. Disponível em: http://www.scielo.br/pdf/ci/ v33n3/a06v33n3.pdf

26. Emirbayer M, Goodwin J. Network analysis, culture, and the problem of agency. Am J Sociol. 1994;99(6):1411-53. Available from: http://as.nyu.edu/docs/IO/233/ais94.pdf

27. Peduzzi M. Equipe multiprofissional de saúde: conceito e tipologia. Rev Saúde Pública. 2001;35(1):103-9. http://dx.doi. org/10.1590/S0034-89102001000100016.

28. Schraiber LB, D’Oliveira AF, Hanada H, Kiss L. Assistência a mulheres em situação de violência - da trama de serviços à rede intersetorial. Athenea Digital. 2012;12(3):1-24. Disponível em: http://psicologiasocial.uab.es/athenea/index. php/atheneaDigital/article/view/ 1110

29. Kiss LB, Schraiber LB, D’Oliveira AFPL. Possibilidades de uma rede intersetorial de atendimento a mulheres em situação de violência. Interface Comun Saúde Educ. 2007;11(23):485501. http://dx.doi.org/10.1590/S1414-32832007000300007 
D’Oliveira AFPL, Schraiber LB. Mulheres em situação de violência.

30. Colombini M, Mayhew S, Watts C. Health-sector responses to intimate partner violence in low and middle- income settings: a review of current models, challenges and opportunities. Bull World Health Org. 2008;86(8):635-42. doi: 10.2471/ BLT.07.045906

31. Brasil. Ministério da Saúde. Violência intrafamiliar orientações para a prática em serviço. Brasília; 2001. (Caderno de Atenção Básica, n.8). Disponível em: http://bvsms.saude. gov.br/bvs/publicacoes/cd05_19.pdf

32. Kiss LB, Schraiber LB. Temas médico-sociais e a intervenção em saúde: a violência contra mulheres no discurso dos profissionais. Ciênc Saúde Coletiva. 2011;16(3):1943-52. Disponível em: http://www.scielo.br/pdf/csc/v16n3/28.pdf
33. Okabe I, Fonseca RMGS. Violência contra a mulher: contribuições e limitações do sistema de informações. Rev Esc Enf USP. 2009;43(2):453-8. http://dx.doi.org/10.1590/ S0080-62342009000200027

34. Brasil. Secretaria de Políticas paras as Mulheres. Rede enfrentamento à violência contra a mulher. Disponível em: https://sistema3.planalto.gov.br//spmu/atendimento/ atendimento_mulher.php

35. D'Oliveira AFPL, Schraiber LB, Hanada H, Durand JG. Atenção integral à saúde de mulheres em situação de violência de gênero: uma alternativa para a atenção primária em saúde. Cien Saude Coletiva. 2009;14(4):1037-50. http://dx.doi. org/10.1590/S1413-81232009000400011 